\title{
El potencial para el desarrollo inteligente de las áreas rurales. Los clústeres espaciales de actividades creativas en España'
}

\author{
Ana Isabel Escalona-Orcao ${ }^{2}$ y Severino Escolano-Utrilla ${ }^{3}$
}

\begin{abstract}
RESUMEN
Las actividades creativas, concretamente las Ilamadas "creaciones funcionales", son reconocidas internacionalmente como instrumentos del crecimiento territorial inteligente. Asumiendo que la viabilidad de dicha estrategia en las pequeñas ciudades y áreas rurales aumenta cuando existen clústeres de actividades creativas, hemos utilizado técnicas geoestadísticas para detectar clústeres espaciales o conjuntos de municipios próximos entre cuyas empresas creativas sí podrían generarse, o al menos emularse, las interrelaciones y beneficios propios de los grandes clústeres empresariales urbanos. En un área de estudio inusualmente extensa y manteniendo un elevado nivel de resolución, se han identificado 115 clústeres creativos, el $75 \%$ de los cuales acreditan niveles de especialización suficientes para ser competitivos y dinamizar el territorio. Estos resultados parecen avalar la aplicación de estrategias de desarrollo inteligente en las áreas estudiadas y la recomendación de que las políticas de desarrollo local las incorporen aprovechando la presencia creciente de actividades creativas en el ámbito rural.
\end{abstract}

Palabras clave: Actividades creativas, creaciones funcionales, clusters espaciales, desarrollo rural, desarrollo inteligente

\begin{abstract}
The creative activities, particularly the so-called "functional creations", are internationaIly recognized as instruments of territorial smart growth. Assuming that the feasibility of the strategy in small towns and rural areas increases when there are clusters of creative activities, we have applied geostatistical techniques to detect spatial clusters or groups of neighboring municipalities within which the creative firms can generate or, at least, emulate the interrelations and benefits typical of large urban clusters. In an unusually extensive study area and maintaining a high level of resolution, 115 creative clusters have been identified, $75 \%$ of which appear being specialized enough as to improve the competitivity and dynamism of the territory. The results seem to support the viability of smart growth strategies in the areas studied and the recommendation that local development policies should incorporate them taking advantage of the growing presence of creative activities in rural areas.
\end{abstract}

Key words: Creative activities, functional creations, spatial clusters, rural development, smart growth

Artículo recibido el 9 de enero de 2016, aceptado el 11 de marzo de 2016 y corregido el 11 de mayo de 2016

Departamento de Geografía y Ordenación del Territorio, Instituto Universitario de Ciencias Ambientales, Universidad de Zaragoza (España). E-mail: aescalon@unizar.es

3 Departamento de Geografía y Ordenación del Territorio, Instituto Universitario de Ciencias Ambientales, Universidad de Zaragoza (España). E-mail: severino@unizar.es 
Este artículo estudia la localización de clústeres espaciales de actividades creativas en las áreas rurales. ${ }^{4}$ La valoración de estas actividades como herramientas para el desarrollo territorial no ha parado de crecer. Por todo ello instituciones internacionales como la Unión Europea o la OCDE consideran que las actividades creativas pueden favorecer el crecimiento inteligente, sostenible e inclusivo a todas las escalas y recomiendan reforzar su potencial para, de este modo, promover y hacer extensivos sus beneficios al conjunto del territorio (European Commission, 2012; OECD, 2013). Esta determinación resulta muy apropiada para las pequeñas ciudades y las áreas rurales que siguen presentando, en general, una situación de menos desarrollo respecto de las grandes ciudades y áreas urbanas.

El impulso a las actividades creativas como herramienta de desarrollo inteligente en el ámbito rural supone hacer frente a diversas imprecisiones. Naldi et al. (2015) han señalado que todavía no está claro su encaje en unas áreas tan complejas y heterogéneas y reclaman estudios sobre posibles factores determinantes del crecimiento inteligente en estas áreas con el fin de establecer el potencial que les confiere. El presente trabajo sale al paso de esa demanda proponiendo una metodología para la localización de posibles clústeres espaciales de actividades creativas, y concretamente de las denominadas "creaciones funcionales" -arquitectura, ingeniería, publicidad, informática, programación- en las áreas rurales españolas. El tema es oportuno porque investigaciones recientes coinciden en señalar que la existencia de una economía creativa puede sustentar el crecimiento inteligente en las áreas rurales (Naldi et al., 2015; Jakob y Van Heur, 2015). Ocurre además que las actividades que integran el sector de las creaciones funcionales son reconocidas como una parte altamente innovadora de la economía creativa (Mateos García et al., 2014; Chapain et al., 2010; Bakhshi et al., 2015).

Las preguntas que nos planteamos son las siguientes: ¿Qué importancia tienen las actividades consistentes en creaciones funcionales en las áreas rurales? ¿Qué posibles clústeres espaciales existen y dónde se localizan? ¿Qué valoración puede hacerse de los mismos en relación con el potencial local para el crecimiento inteligente? Para darles respuesta manejamos información detallada sobre el número de empresas y de empleos en el sector de las creaciones funcionales en 7.367 municipios españoles de menos de 50.000 habitantes situados fuera de las áreas urbanas del país y aplicamos técnicas geoestadísticas para detectar concentraciones significativas, clústeres espaciales, de estas actividades. La extensa área del estudio, más del $90 \%$ del territorio español, y el elevado nivel de resolución espacial al que se lleva a cabo, el municipio, representa una importante aportación del trabajo ya que en este ámbito dominan los estudios de caso. También queremos destacar la eficacia de la metodología aplicada, que nos ha permitido identificar 115 clústeres espaciales de diverso grado de densidad empresarial. Además, y en relación con la tercera pregunta, hemos verificado que algo más del $75 \%$ de los clústeres espaciales están especializados en actividades creativas, atributo que refuerza su potencial para estimular un desarrollo inteligente en sus territorios de implantación.

El resto del artículo queda como sigue: en el segundo apartado recogemos los antecedentes teóricos; en el tercero explicamos la metodología aplicada; en el cuarto describimos y analizamos los clústeres espaciales detectados y su potencial para estimular un desarrollo inteligente en las

La investigación que da origen al trabajo está siendo financiada por el Ministerio de Economía y Competitividad del Gobierno de España (código de proyecto: CSO2O12-31650) 
áreas rurales de su entorno y en la conclusión evaluamos los resultados obtenidos y proponemos nuevas líneas de investigación.

\section{Los clústeres espaciales de actividades creativas como vía para un desarrollo rural inteligente. Problemática, principios y supuestos}

La compleja situación de muchas áreas rurales y su difícil convergencia con el nivel de rentas y de bienestar de las áreas urbanas, justifica la búsqueda de nuevas fórmulas para impulsar su desarrollo territorial. En el caso concreto de las áreas rurales españolas, y de las del interior en particular, su declive demográfico, social y económico requiere identificar qué posibles dinámicas innovadoras existen y hasta qué punto pueden contribuir a paliar una decadencia estructural (Bayer y Baumgartner, 2014; Escalona et al., 2016 y 2015). En esta línea se enmarca la posible inclusión de las áreas rurales entre las potenciales beneficiarias de las estrategias de especialización inteligente. Es oportuno recordar que esta estrategia consiste en identificar y aprovechar las ventajas competitivas del territorio en términos de conocimiento e innovación (Barca et al., 2012). La crisis económica actual ha puesto de manifiesto una necesidad clara de distribución prudente de los recursos financieros disponibles para estimular el desarrollo territorial, tanto a nivel nacional y regional como local (McCann y Ortega-Arguilés, 2015). Por ello la lógica que sustenta la estrategia consiste en concentrar los esfuerzos en un número limitado de actividades consideradas como prioritarias, para permitir a los territorios adquirir, y mantener, altos niveles de competitividad en una economía global (McCann y Ortega-Argilés, 2015; European Commission, 2012). Ahora bien, ¿pueden trasladarse los principios de la especialización inteligente al heterogéneo conjunto espacial que constituyen las áreas rurales? Naldi et al. (2015) han señalado que todavía no está claro y reclaman estudios específicos sobre los posibles factores determinantes del crecimiento rural inteligente. Mientras tanto aportan algunas hipótesis relativas a circunstancias que podrían resultar determinantes $\mathrm{o}$, al menos, favorecerlo. La primera es que el crecimiento inteligente sería mayor en aquellas áreas rurales que se asemejan a las urbanas y en las que, por tanto, pueden desarrollarse economías de aglomeración. Esta hipótesis viene avalada por la evidencia de que las economías de aglomeración, y en concreto, el desarrollo y la difusión de innovaciones surgen en las áreas urbanas como consecuencia de las relaciones entre las empresas que se en ellas se concentran y constituyen clústeres.

La segunda hipótesis establece que el crecimiento inteligente sería más probable en las áreas capaces de desarrollar una economía creativa. Esta hipótesis se ampara en una abundante bibliografía sobre la presencia y efectos en las áreas rurales de las llamadas actividades creativas, es decir, las que generan "...productos tangibles o servicios intangibles -intelectuales o artísticoscon contenido creativo, valor económico y objetivos de mercado" (UNCTAD, 2010: 7) dirigidos a una demanda amplia, diversificada y creciente (Enders y Hayes, 2014). Se trata de un conjunto heterogéneo en el que se incluyen desde las actividades arraigadas en la cultura tradicional - Patrimonio, Artes- a las que elaboran contenidos creativos para grandes audiencias -incluidas en el ámbito de la Comunicación-, pasando por las consideradas como "creaciones funcionales", es decir, aquellas que proporcionan bienes y servicios (software, publicidad, ingeniería, consultoría, arquitectura) con un elevado contenido creativo (UNCTAD, 2010; Méndez et al., 2012). Como ya se ha dicho, a las actividades que integran el grupo de creaciones funcionales se les conoce por su 
propensión a innovar (Mateos García et al., 2014) y su elevada intensidad del conocimiento, vectores básicos del crecimiento inteligente, lo que reafirma su idoneidad como posibles detonantes del mismo y justifica que centremos en ellas el presente análisis.

Aunque las actividades creativas se localizan preferentemente en grandes ciudades cada vez son más frecuentes fuera de ellas (Bell y Jayne, 2010; Vallance, 2014; Escalona et al., 2016). En los pequeños municipios cercanos a grandes áreas urbanas encontramos free-lancers y pequeñas empresas creativas que ponderan positivamente la proximidad a un gran mercado (De Propris et al., 2009). Además las mejoras en las tecnologías de la información y la comunicación han brindado opciones para la localización de estas actividades en áreas más remotas que, de este modo, han podido sacar partido de otros atractivos como la calidad de vida que ofrecen. De hecho ya hace algún tiempo que en estudios realizados en los Estados Unidos y en Europa se demostró cómo, en los casos de localidades remotas pero con buena conectividad, las llamadas amenidades -recursos naturales y culturales para el disfrute de los residentes y visitantes- atraen a las clases creativas, es decir, a trabajadores muy cualificados, especializados en estas actividades $y$, por tanto, impulsores de las mismas en las localidades en las que se asientan (Mcgranaham et al., 2007 y 2010; Lafuente et al., 2010).

Matizando lo expuesto, Anderson et al. (2015) han señalado recientemente que si bien las áreas rurales son un buen lugar para que surja la creatividad no lo son tanto para su aplicación en contextos empresariales. Es una idea interesante que justifica la presencia de emprendedores en localidades pequeñas pero plantea la dificultad estructural de que se consoliden entornos productivos que puedan estimular el desarrollo local. De hecho, en la mayor parte de los municipios rurales, pequeños y dispersos, no es fácil que surjan concentraciones, o clústeres, de empresas que compartan experiencias y conocimientos $y$, de este modo, propicien las innovaciones y las demás externalidades propias de un cluster (Porter, 1998; Spencer et al., 2010; Rodríguez-Pose y Comptour, 2012; Titze et al., 2014). Una masa crítica de actividad creativa, susceptible de sustentar un crecimiento territorial inteligente, se alcanzaría únicamente sumando las empresas de un cluster espacial, es decir, de un conjunto de municipios próximos entre cuyas empresas creativas sí podrían generarse interrelaciones y economías de aglomeración. Este planteamiento queda implícito en diversos trabajos anteriores sobre la geografía de las actividades creativas en las áreas rurales, en los que se utilizan como unidades de análisis demarcaciones supramunicipales de diversos tipos, como los mercados de trabajo (Lazeretti et al., 2008; Boix y Lazeretti, 2012) o las Ilamadas travel to work areas (De Propris et al., 2009). Nuestra aproximación a la identificación de clústeres espaciales es distinta ya que no partimos de demarcaciones preestablecidas sino que se delimitan como resultado de la metodología que explicamos seguidamente.

\section{Datos y metodología del análisis}

Para identificar clústeres espaciales de actividades creativas susceptibles de impulsar estrategias de desarrollo inteligente en las áreas rurales, necesitamos disponer de datos apropiados, exhaustivos y al máximo nivel de detalle espacial. Por esta razón, descartadas las bases de microdatos empresariales por su deficiente cobertura del medio rural, manejamos los datos municipales proporcionados por la Tesorería General de la Seguridad Social y, en concreto, el número de empresas en los sectores creativos pertenecientes a la categoría de "creaciones funcionales". Los epígrafes de la Clasificación Nacional de Actividades Económicas (CNAE) de 2009 que mejor encajan en el concepto de creaciones funcionales ya expuesto se presentan en Cuadro № 1. 
Cuadro No 1

Actividades consideradas como creaciones funcionales

\begin{tabular}{|c|c|}
\hline \multirow{5}{*}{ Creaciones funcionales } & 74. Otras actividades profesionales, científicas y técnicas (1) \\
\hline & $\begin{array}{l}\text { 71. Servicios técnicos de arquitectura e ingeniería; ensayos y análisis } \\
\text { técnicos }\end{array}$ \\
\hline & 73. Publicidad y estudios de mercado \\
\hline & $\begin{array}{l}\text { 70. Actividades de las sedes centrales; actividades de consultoría de } \\
\text { gestión empresarial (2) }\end{array}$ \\
\hline & $\begin{array}{l}\text { 62. Programación, consultoría y otras actividades relacionadas con } \\
\text { la informática }\end{array}$ \\
\hline
\end{tabular}

Fuente: Elaboración propia a partir de UNCTAD, 2010.

(1) Este subsector incluye las actividades de diseño especializado, traducción e interpretación. (2) Este subsector incluye las actividades de relaciones públicas y comunicación

El área de estudio es la que suman todos los municipios de menos de 50.000 habitantes situados fuera de las grandes áreas urbanas delimitadas oficialmente (Ministerio de Fomento, 2006). De este modo el estudio se ampara en un criterio riguroso para la identificación del ámbito rural y cubre áreas pendientes de este tipo de análisis. Son 7.367 municipios en total, que representan el $90,8 \%$ de los municipios españoles, albergan el $32,2 \%$ de la población total y abarcan el $90,7 \%$ de la superficie del país.

La metodología desarrollada cumple estos dos requisitos: es coherente con nuestro concepto de cluster espacial -conjunto de municipios próximos entre cuyas empresas creativas se generan interrelaciones y economías de aglomeración- y capta dos atributos clave del mismo: sus límites y la densidad de masa empresarial. Existe una amplia gama de técnicas de estadística espacial -índice de Getis-Ord, índice local de Moran, densidad kernel, índice del vecino más próximo, análisis de agrupamientos, entre otras- que identifican concentraciones espaciales de elementos o unidades espaciales de un fenómeno determinado a las que se denomina clústeres, hot spots o de forma similar. Son técnicas que incorporan en el cómputo la estructura espacial de las unidades observadas - municipios en nuestro caso- y los posibles efectos de interacción entre dichas unidades basados en la vecindad o en otra medida de la distancia (Cromley y Hanink, 2012). Esta cualidad es oportuna para nuestro trabajo por la probabilidad de que en el área estudiada se manifiesten fenómenos de autocorrelación espacial, de modo que la presencia e intensidad de las actividades creativas en un lugar aparezca relacionada con la de las localidades vecinas. La hipótesis de la autocorrelación vendría reforzada, además, por las interrelaciones empresariales implícitas en todo cluster.

El procedimiento aplicado para identificar clústeres espaciales de actividades creativas se diferencia de otros previos (Boix et al., 2011; Bertacchini y Borrione, 2013; Villarreal y Flórez, 2015; Boal y Herrero, 2015) porque combina dos de las técnicas antes citadas: el cálculo de la densidad focal mediante la función kernel y el análisis de agrupamientos. El cálculo de la densidad focal encaja en nuestro concepto de cluster espacial porque, como es sabido, permite mostrar el reparto de la masa de empresas creativas por el territorio sin las distorsiones derivadas de la desigual extensión de las unidades espaciales de referencia, municipios en este caso. En su aplicación hemos adoptado los siguientes parámetros: un tamaño de pixel con resolución de $1 \mathrm{~km}$. y un ancho de 
banda de $30 \mathrm{kms}$. El análisis de agrupamientos, por su parte, permite delimitar el cluster espacial, es decir, determinar el conjunto de municipios que lo integran. Los parámetros adoptados en este caso son: un número mínimo de municipios de 5 o más y una distancia máxima entre los mismos de $30 \mathrm{kms}$. Todos los parámetros seleccionados se adecuan a las peculiaridades del caso. Como veremos en el siguiente apartado solo 1.633 municipios del área de estudio tienen empresas creativas en los sectores de creaciones funcionales. Se trata, además, de una distribución notablemente asimétrica ya que aunque el número máximo de empresas en un municipio es de 155, la moda de la distribución es 1 (42'4\% de los municipios tienen solo una empresa) y la mediana, 2.

En el contexto territorial propio del caso expuesto entendemos, y hemos verificado, que el tamaño de pixel elegido -que genera una malla de 921 filas y 1.137 columnas al cubrir todo el territorio peninsular e insular- es adecuado para el análisis de la distribución espacial de las empresas. El número mínimo de municipios, 5, minimiza el riesgo de que la masa empresarial reunida no baste para generar economías de escala. Por su parte, la distancia de $30 \mathrm{kms}$. elegida para el ancho de banda y establecer la separación intermunicipal, supera ampliamente la distancia media entre los municipios, 9,2 kilómetros, y resulta apropiada para describir el patrón habitual de los probables movimientos pendulares resultantes de las interrelaciones empresariales típicas de un cluster. Cabe añadir que la distancia adoptada coincide con la propuesta de forma automática por el software utilizado -Crimestat IV, hot spot analysis- y que, en todo caso, el manejo de valores distintos no alteraría sustancialmente los resultados (Moreno, 1991).

Las dos técnicas descritas -cálculo de la densidad focal y análisis de agrupamientos- se han aplicado de forma sucesiva para delimitar clústeres espaciales de municipios con empresas de creaciones funcionales con diversos niveles de densidad en su masa empresarial.

\section{Los clústeres espaciales de creaciones funcionales en las áreas rurales españolas}

Los datos del Cuadro No 2 dan respuesta a la primera de las preguntas planteadas en este trabajo ya que reflejan la importancia de las creaciones funcionales en la amplia área de estudio.

Cuadro No 2

Presencia de empresas de creaciones funcionales en los municipios del área de estudio

\begin{tabular}{|l|r|r|r|}
\hline \multirow{2}{*}{ Área de estudio } & \multicolumn{2}{|c|}{$\begin{array}{r}\text { Municipios con empresas de creaciones } \\
\text { funcionales }\end{array}$} \\
\cline { 3 - 4 } & & \multicolumn{1}{|c|}{ Total } & \% área de estudio \\
\hline No de municipios & 7.367 & 1.633 & 22,16 \\
\hline Población & 14.985 .859 & 11.223 .425 & 74,89 \\
\hline Empresas (total) & 564.349 & 437.837 & 77,58 \\
\hline Empleos (total) & 4.090 .733 & 3.184 .169 & 77,8 \\
\hline Empresas de creaciones funcionales & 7.582 & 7.582 & 100 \\
\hline Empleos en creaciones funcionales & 56.819 & 52.836 & 99,96 \\
\hline
\end{tabular}

Fuente: Elaboración propia a partir de datos de Censo de la población, 2011 y Tesorería General de la Seguridad Social, 2012. 
Los datos aportados muestran, en primer lugar, que las actividades consistentes en creaciones funcionales representan todavía una parte muy pequeña de las economías de los municipios estudiados, ya que apenas superan el 1,3\% de sus empleos y de sus empresas. Por otra parte están presentes solo en el $22,16 \%$ de los municipios, donde muestran un peso económico ligeramente superior ya que su proporción respecto de las empresas y empleos totales es del 1,7 y 1,6\% respectivamente. Esta elevada selectividad espacial se relaciona con que más de los dos tercios de los municipios que integran el área de estudio tienen menos 1.000 habitantes (Cuadro No 3). Se trata de municipios que aún no se han recuperado de la sostenida e intensa despoblación que vació durante el pasado siglo muchas áreas rurales del interior de España dejándolas con poblaciones extremadamente bajas (Zúñiga et al., 2012).

Cuadro No 3

Distribución por tamaño municipal de los municipios y de las empresas de creaciones funcionales

\begin{tabular}{|c|c|c|c|c|}
\hline \multirow[t]{2}{*}{ Población municipal } & \multicolumn{2}{|c|}{$\begin{array}{l}\text { Municipios del área de } \\
\text { estudio }\end{array}$} & \multicolumn{2}{|c|}{$\begin{array}{c}\text { Empresas de creaciones } \\
\text { funcionales }\end{array}$} \\
\hline & Total & $\%$ & Total & $\%(1)$ \\
\hline$<1.000$ & 4.856 & 65,92 & 290 & 3,8 \\
\hline $1.000<$ población $<5.000$ & 1.740 & 23,62 & 1.420 & 18,7 \\
\hline $5.000<$ población< $<10.000$ & 412 & 5,59 & 1.281 & 16,8 \\
\hline $10.000<$ población<50.000 & 359 & 4,87 & 4.591 & 60,5 \\
\hline
\end{tabular}

Fuente: Elaboración propia a partir del Censo de la población, 2011 y Tesorería General de la Seguridad Social, 2012.

(1) Respecto del total de empresas de creaciones funcionales

La relación entre el número de empresas de creaciones funcionales y el tamaño demográfico de los municipios es clara de modo que el coeficiente de correlación de Pearson toma el valor 0,758 ( $p$-valor $=0,000$ ). Las pequeñas ciudades de más de 10.000 habitantes, donde se concentra la mayoría de las empresas, tienen ventajas para atraer este tipo de actividades debido al tamaño de sus mercados de trabajo, a su potencial para que las empresas se relacionen con otras, y a la existencia de una demanda local de los bienes y servicios que ofrecen este tipo de empresas (Escalona et al., 2015b). Sin embargo la relación con el tamaño demográfico no es lineal ya que en los municipios que tienen entre 5 y 10.000 habitanes la proporción de empresas de creaciones funcionales es menor que en la categoría de 1.000 a 5.000. Este dato es interesante porque suscita la posibilidad de relacionar la presencia de estas empresas en municipios pequeños con el hecho de que tales municipios desempeñen funciones especializadas en su área de influencia. No puede descartarse tampoco que los pequeños municipios con un número desproporcionadamente alto de empresas de creaciones funcionales se encuentren próximos a grandes áreas urbanas, como se aprecia en el mapa de la Figura № 1.

El mapa elaborado muestra, en primer lugar, un notable contraste entre los espacios rurales de las regiones periféricas y merididionales, por una parte, y los de las regiones del interior. En los primeros hay mayor densidad de municipios con existencia de estas empresas $y$, además, sus empresas de creaciones funcionales son más numerosas. Queda clara también, como ya se ha indicado, una notable acumulación de estos municipios más creativos en los entornos de las principales áreas metropolitanas (Bilbao-S. Sebastian, Barcelona, Valencia-Alicante, Sevilla, Ma- 
Figura No 1

Distribución espacial de los municipios del área de estudio con empresas de creaciones funcionales

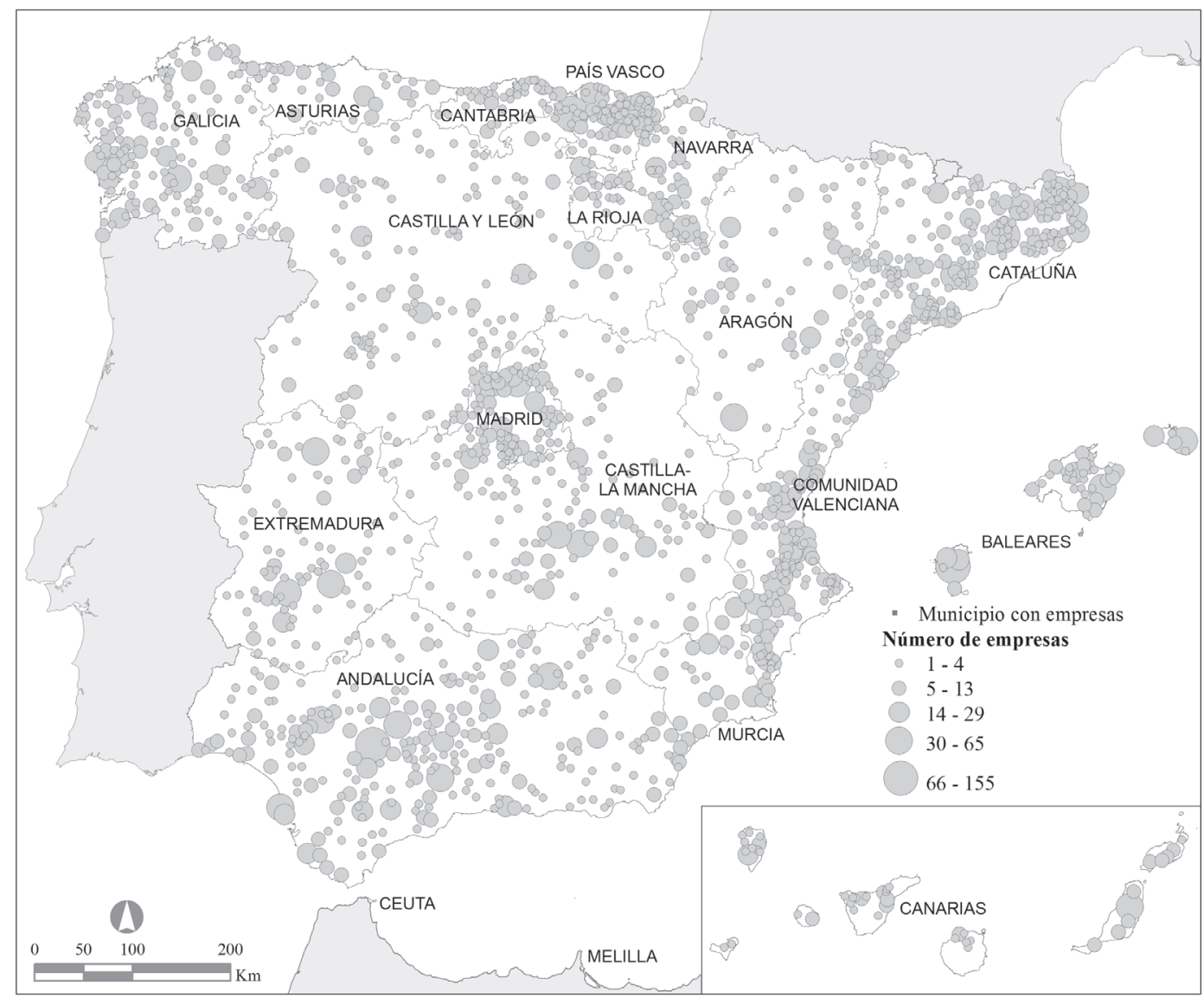

Fuente: Elaboración propia a partir de Tesorería General de la Seguridad Social, 2012.

drid) y en la costa de Galicia. Este resultado confirma las expectativas respecto de las ventajas de localización en pequeñas localidades próximas a los mercados urbanos y metropolitanos. Sin embargo en las regiones del interior, los municipios con este tipo de empresas son menos y a ello se añade su mayor dispersión y menor concentración de empresas. Sin duda este patrón espacial va a resultar clave para la localización y distribución de los clústeres espaciales, como exponemos a continuación.

\section{Los clústeres espaciales de empresas de creaciones funcionales. Identificación y localización}

Con este apartado damos respuesta a la segunda de las preguntas planteadas. Siguiendo la metodología establecida hemos obtenido en primer lugar la densidad por kilómetro cuadrado de las 7.582 empresas de creaciones funcionales existentes en los 1.633 municipios con una o más de dichas empresas (Figura No 2). 
Figura No 2

Densidad de las empresas de creaciones funcionales en la zona de estudio

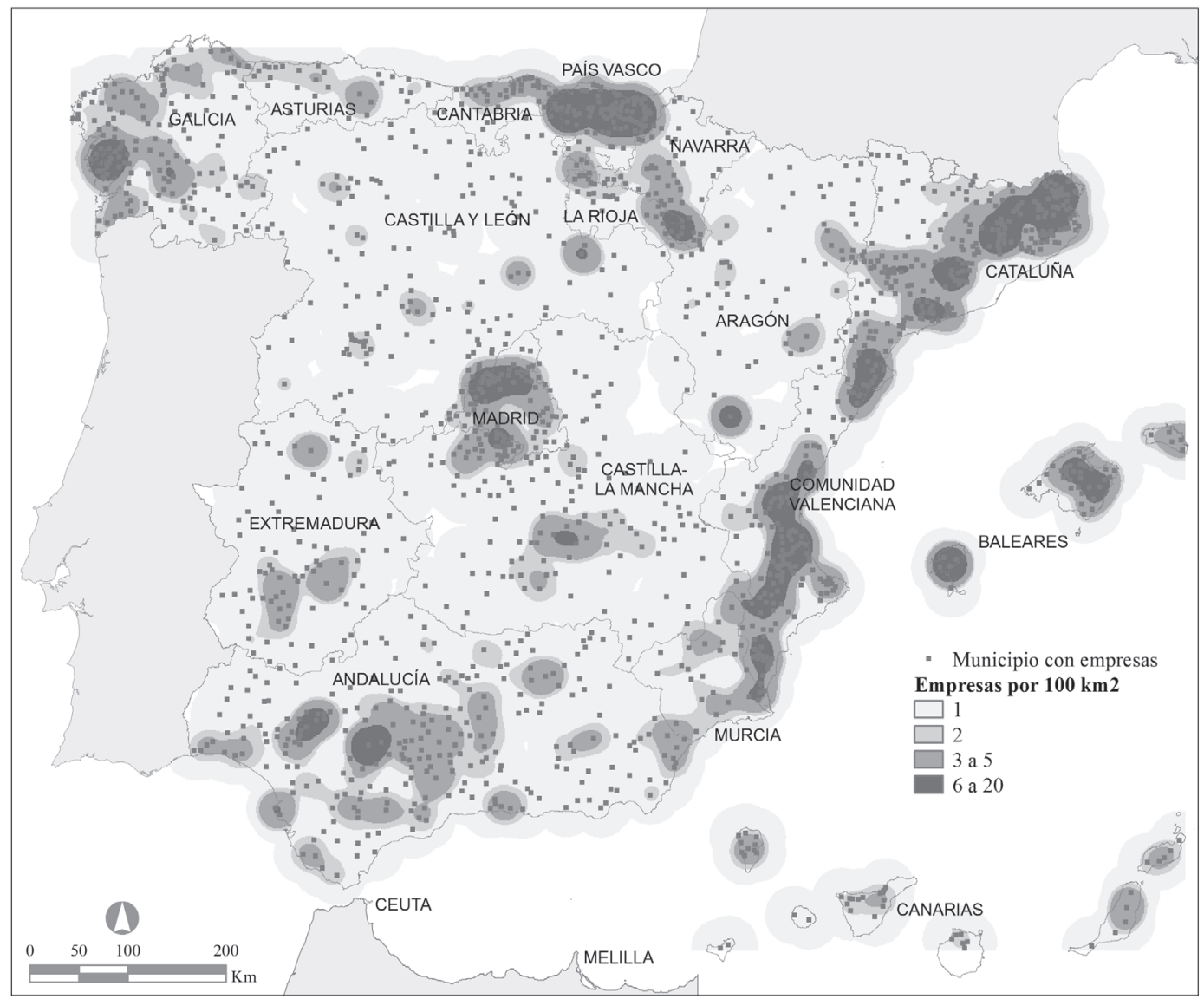

Fuente: Elaboración propia a partir de Tesorería de la Seguridad Social.

El mapa resultante de aplicar el método de la densidad focal (kernel) resulta muy expresivo ya que realza los contrastes espaciales presentes en el mapa de la Figura № 1. Las mayores densidades de empresas se circunscriben a los entornos de las grandes aglomeraciones urbanas de la costa mediterránea (hasta Murcia), País Vasco, Galicia (Vigo) y Madrid. Son Ilamativas también las concentraciones que aparecen en el interior de Andalucía, la parte alta del Valle del Ebro y la Cataluña Oriental. En el resto del territorio la densidad empresarial es extremadamente baja, lo que justifica la necesidad de identificar clústeres espaciales de municipios que puedan reunir una masa crítica susceptible de poner en marcha procesos de desarrollo inteligente. Para ello hemos aplicado los criterios de agrupamiento establecidos, obteniéndose 115 agrupamientos o clústeres espaciales integrados por 1.301 municipios. Al superponer el mapa obtenido con el de las densidades focales, y categorizar estas últimas en tres niveles, hemos obtenido la tipología de clústeres espaciales que se describe en el cuadro $N^{\circ} 4$.

Los clústeres más numerosos son los que contienen una densidad de empresas creativas baja. Este resultado es coherente con las características de la zona de estudio comentadas en relación 
Cuadro No 4

Características de los clústeres espaciales de actividades consistentes en creaciones funcionales

\begin{tabular}{|c|c|c|c|c|c|c|c|}
\hline \multirow{2}{*}{$\begin{array}{l}\text { Tipo de cluster } \\
\text { según densidad (1) }\end{array}$} & \multirow{2}{*}{$\begin{array}{l}\text { No de } \\
\text { clústeres }\end{array}$} & \multirow{2}{*}{$\begin{array}{c}\mathrm{N}^{\circ} \mathrm{de} \\
\text { municipios }\end{array}$} & \multirow{2}{*}{$\begin{array}{l}\text { Población media } \\
\text { municipal }\end{array}$} & \multicolumn{4}{|c|}{$\begin{array}{c}\text { Número de municipios } \\
\text { por tamaño (2) }\end{array}$} \\
\hline & & & & $A$ & $B$ & $C$ & $\mathrm{D}$ \\
\hline Baja $(<2)$ & 56 & 451 & 5.245 & 74 & 199 & 92 & 67 \\
\hline Media $(2<$ densidad $<5$ & 36 & 402 & 7.289 & 44 & 176 & 91 & 91 \\
\hline Alta $(>5)$ & 23 & 448 & 8.180 & 55 & 178 & 108 & 126 \\
\hline Todos & 115 & 1.301 & 6.937 & 173 & 553 & 291 & 284 \\
\hline
\end{tabular}

Fuentes: Elaboración propia a partir de Tesorería General de la Seguridad Social y Censo de la población 2011. (1) Empresas/100 km. (2) A (pob < 1.000), B (1000< pob < 5.000), C (5.000< pob < 10.000), D (pob $>10.000)$

con el mapa de densidad. El reparto de los 1.301 municipios entre los tres tipos de clústeres es equilibrado, si bien los clústeres de baja densidad se distinguen por la mayor presencia de municipios pequeños entre sus integrantes. La población media de los clústeres menos densos multiplica por 2,7 la media de los municipios de la zona de estudio lo que da un indicio del aumento de masa crítica que aporta el agrupamiento realizado. Los pequeños municipios también aparecen formando parte de los clústeres de densidad media y alta, aunque está claro que estos se establecen en torno a los de mayor tamaño. De ahí deriva su mayor población media que cuadruplica la de la zona de estudio.

La localización espacial de los tres tipos de clústeres se detalla en el mapa de la Figura № 3. Los hemos representado mediante elipses de una desviación estándar ya que, aunque abarcan solo en torno al $60 \%$ de los municipios incluidos en los clústeres, esta técnica recoge bien la tendencia direccional del agrupamiento. Además el trazado tiene en cuenta la distinta importancia de los municipios según su número de empresas de creaciones funcionales, de modo que la elipse toma la dirección de los municipios con más empresas.

Según este mapa encontramos que son clústeres espaciales la mayoría de las áreas destacadas por su densidad empresarial en el mapa anterior (Figura $N^{\circ} 2$ 2). Ello es lógico porque la alta densidad empresarial aparece normalmente aparejada con una también alta densidad municipal, lo que facilita que en esas áreas los municipios cumplan más fácilmente los requisitos de número y distancia mínima establecidos para constituir clústeres espaciales. Hay algunas excepciones, como sería el caso de las pequeñas ciudades de Teruel y Soria, situadas al sur de Aragón y al este de Castilla León respectivamente y cabeceras de sus provincias. La falta de municipios próximos impide que aparezcan en el mapa de clústeres espaciales a pesar de que sus densidades propias sean estimables y superiores a las de muchos de los clústeres detectados.

Los clústeres de alta densidad se alinean de forma paralela a la costa mediterránea, desde la frontera francesa hasta el sur de Alicante, incluidas las islas Baleares. Reconocemos otra alineación aunque mucho más corta junto al litoral vasco. En los demás casos se presentan aislados (sur de Asturias, norte de Ciudad Real) o entrelazados con otros clústeres menos densos (norte de Madrid, sureste de Navarra, entorno de Sevilla). Todos se encuentran en áreas rurales con una alta 
Figura No 3

Los clústeres espaciales de actividades consistentes en creaciones funcionales

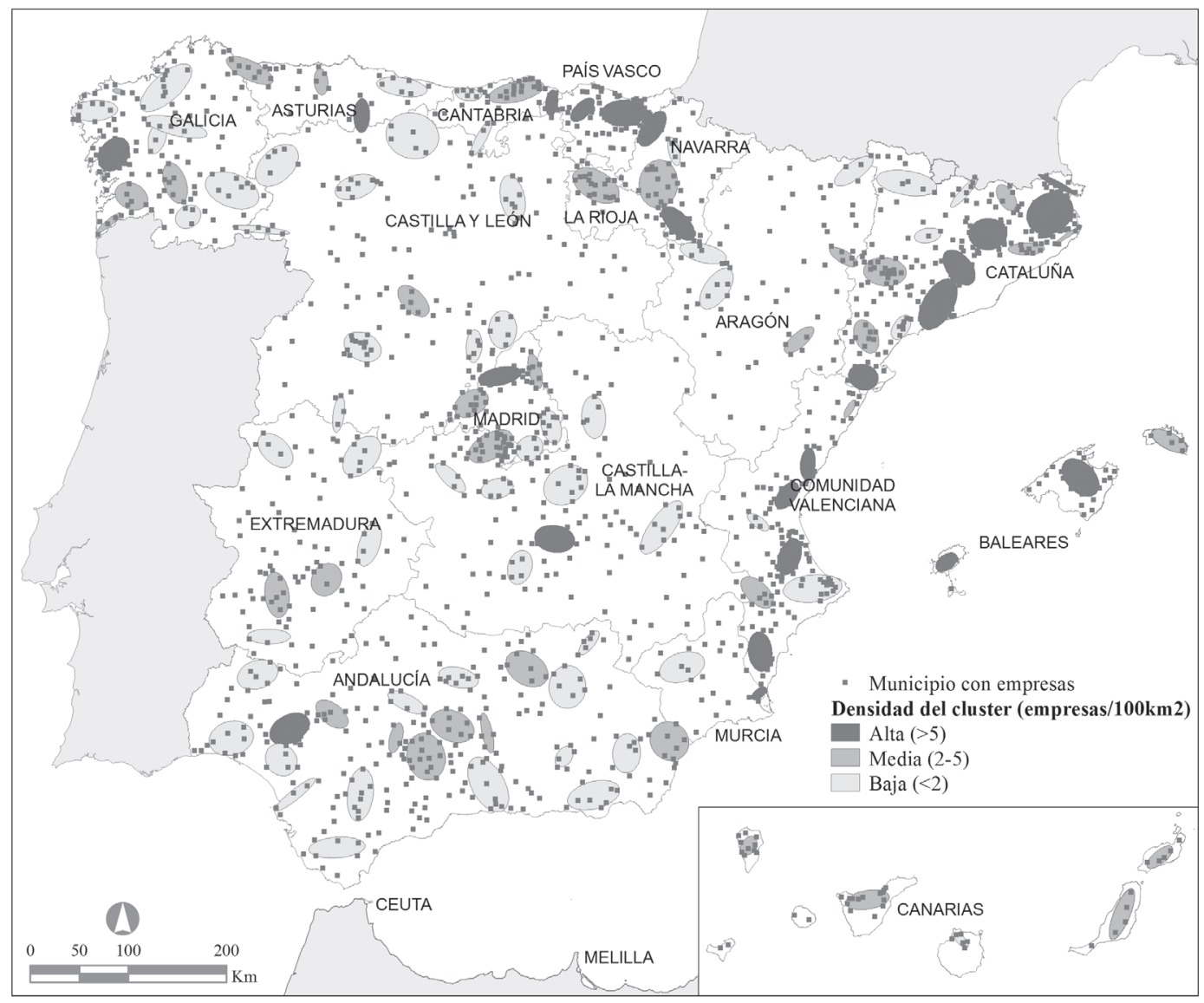

Fuente: Elaboración propia a partir de Tesorería de la Seguridad Social.

intensidad de mercado, debido a la presencia o a la vecindad de industrias y, en menor medida, a la diversificación del tejido empresarial, características que han resultado explicativas en diversos análisis empíricos (Escalona et al., 2016 y 2015). En el resto de la zona de estudio, la vecindad con un área urbana no es suficiente para la existencia de un cluster de alta densidad, lo que explicaría su falta -salvo las excepciones citadas-, en las regiones del interior $y$, sobre todo, al oeste de una diagonal imaginaria entre el sur de Galicia y la costa oriental andaluza. En la amplia área descrita solo encontramos clústeres espaciales de densidad media y baja.

\section{Potencial de los clústeres espaciales para inducir un crecimiento rural inteligente}

La identificación de clústeres espaciales de empresas de creaciones funcionales aparece justificada por la necesidad de encontrar aglomeraciones empresariales susceptibles de impulsar procesos de desarrollo inteligente en su entorno. Este proceso resulta factible en los clústeres más densos, ya que por el tamaño de sus concentraciones empresariales se asemejan a los de las 
áreas urbanas y posibilitan, por tanto, la generación de externalidades positivas (v. apartado 2). En el caso de los clústeres de densidad media y sobre todo en los de densidad baja es oportuno verificar adicionalmente el cumplimiento del segundo de los factores considerados como determinantes para suscitar procesos de desarrollo inteligente, es decir, la especialización en actividades creativas. Para ello hemos obtenido el coeficiente de especialización horizontal de los 115 clústeres espaciales identificados ${ }^{5}$.

Figura No 4

Niveles de especialización de los clústeres espaciales de creaciones funcionales

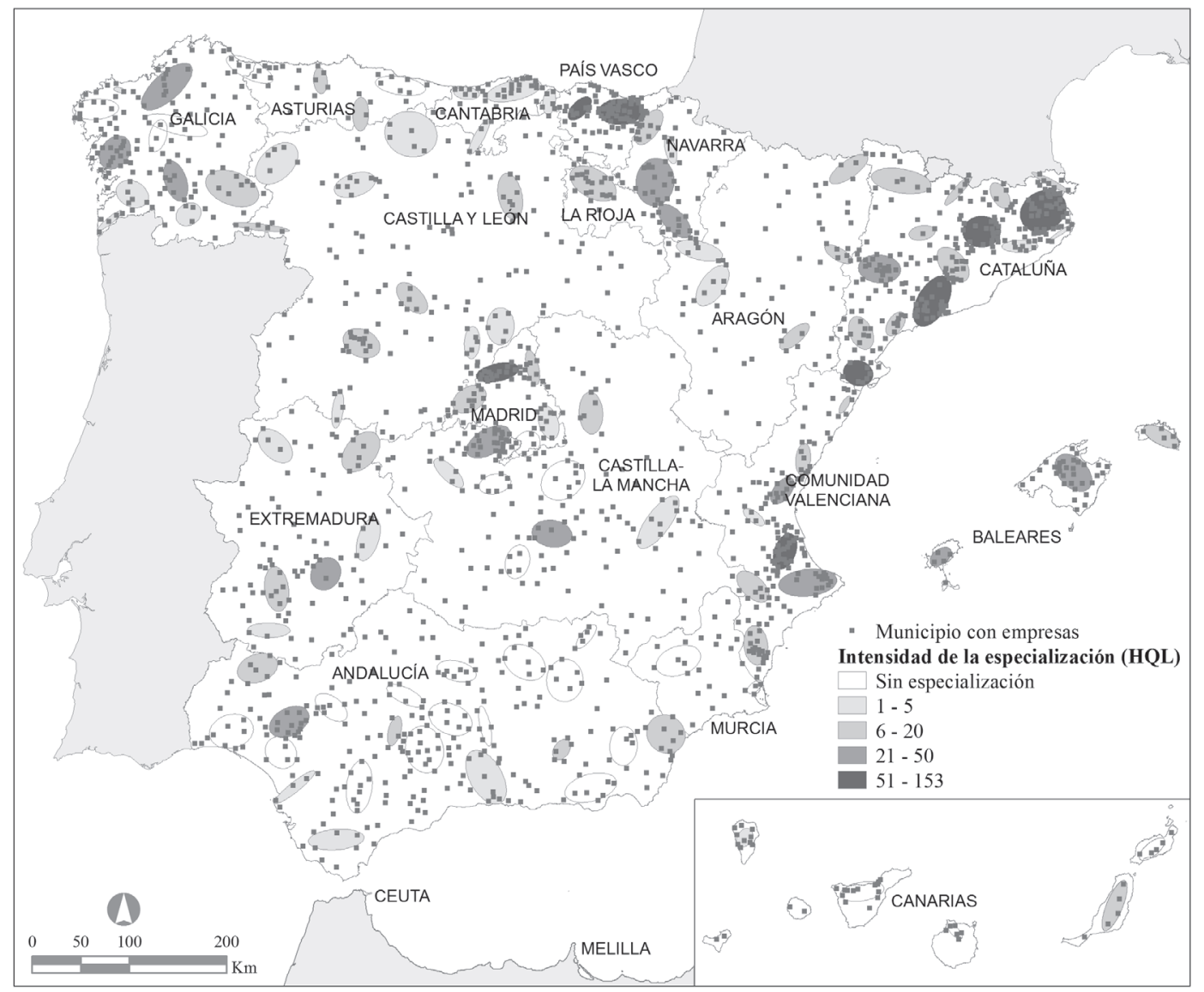

Fuente: Elaboración propia a partir de Tesorería de la Seguridad Social.

Cercano al cociente de localización convencional $(L Q)$, para calcular el cociente horizontal $(H Q L)$ se obtiene primeramente $L Q=(E i j / E j) /(E i / E)$ donde Eij es el número de empresas en la actividad i en la localidad j; Ej, el número total de empresas en la localidad j; Ei, el número total de empresas en la actividad $i$ en el espacio de referencia y $E$, el número total de empresas en dicho espacio. Seguidamente Eij se sustituye por $\hat{E} i j$, de donde resulta $L Q=(\hat{E} i j / E j) /(E i / E)=1$, siendo $\hat{E} i j$ el número de empresas para que $L Q=1$, dados los demás valores. El cociente horizontal se obtiene por último calculando $H L Q=E i j$ - Êij. Valores positivos de HQL son ya indicativos de especialización, aumentando su intensidad conforme lo hace el valor del cociente. 
Los resultados que refleja el mapa de la Figura No 4 apuntan tendencias muy interesantes. En primer lugar solo 27 de los 115 clústeres no están especializados en el sector de creaciones funcionales 0 , a la inversa, en más del $70 \%$ de los clústeres su proporción de empresas de creaciones funcionales les confiere una especialización relativa en dichas actividades. Entre los clústeres especializados encontramos las tres categorías de densidad incluidos los de pequeña y baja densidad. Sin embargo el reparto por el territorio muestra que la mayor parte de los clústeres no especializados están en Andalucía (16), centro peninsular (cuatro entre Castilla la Mancha y Madrid), norte (dos en Asturias y tres en Galicia) y Canarias (dos). Ocurre en estos casos que la media o baja densidad de las empresas de creaciones funcionales en sus clústeres no se compensa con una especialización relativa de los mismos en dichas actividades, lo que suscitaría dudas respecto de su potencial para generar un desarrollo inteligente. En el resto de las regiones y clústeres espaciales detectados sí que se da esta compensación, de modo que los clústeres de baja densidad acreditan especialización de diversa intensidad.

El análisis realizado parece descartar que en buena parte del tercio meridional de España y en alguna zona aislada del litoral asturiano o gallego, los clústeres espaciales de actividades creativas puedan inducir procesos de especialización inteligente por carecer de densidad y de especialización. En cambio la especialización aparece como característica de los clústeres de baja y mediana densidad de amplias áreas del oeste e interior peninsular. Nos parece que este resultado es alentador ya que suscita expectativas de que, a pesar de los problemas estructurales que aquejan a estos territorios, las actividades creativas desarrolladas en los clústeres espaciales detectados puedan ser, al menos, cualitativamente decisivas para actuar como gérmenes de una estrategia de especialización inteligente.

\section{Conclusión}

El presente trabajo afronta el estudio de las actividades creativas como posibles apoyos de estrategias para un desarrollo inteligente en las pequeñas ciudades y áreas rurales. Hemos señalado cómo la noción de desarrollo inteligente impregna las políticas territoriales formuladas recientemente por diversos organismos internacionales y también las dudas que suscita su posible aplicación en espacios distintos de las grandes áreas urbanas y metropolitanas. Siguiendo las indicaciones de Bell y Jane (2010) y Naldi et al., (2015) hemos asumido que el potencial de las áreas rurales para el desarrollo inteligente podría estar asociado a la existencia de concentraciones empresariales, o clústeres, de actividades creativas. Dos motivos explican dicho supuesto: las economías de aglomeración que se generan en los clústeres y que, por tanto, harían extensivas a las áreas rurales - aunque a una escala diferente- las ventajas de tamaño propias de las urbanas; y el carácter especialmente innovador de las actividades creativas y, en concreto, de las incluidas en la categoría de creaciones funcionales.

El pequeño tamaño de la mayoría de los municipios del área de estudio nos ha llevado a adoptar el concepto de cluster espacial, reconociendo así que en la mayor parte de los casos la masa crítica de empresas susceptible de formar un cluster solo se puede reunir sumando las empresas de municipios próximos entre sí. Por ello hemos elaborado una metodología específica, y adaptada a las condiciones de la zona de estudio, para identificar clústeres espaciales de empresas creativas pertenecientes, en concreto, al sector de las creaciones funcionales. Dicha metodología 
combina técnicas de estadística espacial con el cálculo posterior de un índice de especialización relativa (el cociente de localización horizontal). Así, gracias a esta propuesta metodológica hemos podido afrontar algunas de las cuestiones que inspiraban nuestro trabajo y que en investigaciones previas no habían sido atendidas, o de forma muy incompleta.

En primer lugar, hemos verificado que las actividades estudiadas se reparten de forma muy selectiva por el territorio, ya que solo hay empresas de creaciones funcionales en 1.633 de los 7.365 municipios estudiados. Aunque se trata de una proporción baja, su peso en el conjunto resulta equivalente al de algunos de los países occidentales que son líderes en este tipo de actividad (Escalona et al., 2015). Sin embargo casi el $80 \%$ de los municipios con empresas han resultado formar parte de alguno de los 115 clústeres espaciales identificados. Ello se debe a que las áreas con densidad empresarial coinciden grosso modo con las de densidad municipal. Aunque la coincidencia entre ambas distribuciones resulta lógica, no deja de ser destacable porque facilita la formación de los clústeres espaciales y la compensación, por tanto, de la falta de masa crítica empresarial que -como hemos señalado- es típica de municipios como los estudiados en este trabajo.

Hemos ideado una escala de densidad empresarial de los clústeres espaciales en tres niveles, con el fin de graduar su potencial para sustentar estrategias de desarrollo inteligente en el territorio. El escaso número de clústeres con alta densidad empresarial ( $>5$ empresas/100 km²), ha aconsejado verificar si en los de densidad media y baja -con un potencial dudoso para impulsar un desarrollo inteligente en sus respectivos territorios- esta condición se veía compensada por su especialización relativa. En el subsiguiente análisis hemos verificado que el $70 \%$ de los clústeres están especializados en creaciones funcionales y que entre ellos están la mayoría de los de densidad media y muchos de densidad baja. Este resultado nos parece importante porque da oportunidades de sustentar un desarrollo inteligente a muchos clústeres cuya masa empresarial es de menor entidad.

Los mapas aportados establecen una localización desigual y relativamente concentrada de los aspectos estudiados dentro de un territorio tan heterogéneo y desequilibrado como el español. La densidad empresarial y la municipal, coincidentes como se ha dicho, reflejan la influencia de las grandes áreas urbanas, en cuya proximidad encontramos los clústeres espaciales con más densidad de empresas. Esto es así por los efectos positivos que la proximidad a estas áreas generan sobre las actividades estudiadas, ya que proporcionan a las empresas de creaciones funcionales posibles mercados o les facilitan factores de producción específicos. En las regiones del interior los clústeres espaciales apenas tienen continuidad geográfica y aparecen como islas en medio de amplias áreas donde no hay apenas actividad creativa. Son además clústeres de densidad media y, sobre todo, baja. Sin embargo en una proporción estimable de los mismos, la importancia relativa de las empresas de creaciones funcionales supera a la de otros sectores. Esta especialización puede interpretarse como el cumplimiento de un prerrequisito para que puedan generarse y tener éxito estrategias de desarrollo rural inteligente.

En suma, con este trabajo creemos haber demostrado que es posible que surjan clústeres espaciales creativos en pequeñas ciudades y municipios rurales de todo tipo y que, como han acreditado niveles de especialización suficientes para ser competitivos, pueden actuar como elementos dinamizadores del territorio. Nos parece que los resultados expuestos despejan las dudas suscitadas por la viabilidad de las estrategias de desarrollo inteligente en las áreas estudiadas y 
permiten recomendar que las políticas de desarrollo rural las incorporen aprovechando la presencia creciente de actividades creativas en dichas áreas.

\section{Referencias bibliográficas}

ANDERSON, A.; WALLACE, C. \& TOWNSEND, L. Great expectations or Small Country Living? Enabling Small Rural Creative Business with ICT. Sociologia ruralis, 2015, Vol. 56, No 3, p. 450-468.

BAKHSHI, H.; DAVIES J.; FREEMAN, A. \& HIGGS. P. The Geography of the UK's Creative and High Tech Economies. Londres: NESTA, Informe de investigación, 2015.

BARCA, F.; MCCANN, P. \& RODRÍGUEZ-POSE, A. The case for regional development intervention: place-based versus place-neutral intervention. Journal of Regional Science, 2012, Vol. 52, No 1, p. 134-152.

BAYER, H. \& BAUMGARTNER, D. The Role of Entrepreneurship and Innovation in Peripheral Regions. The Planning Review, 2014, Vol. 50, No 1, p. 16-23.

BELL, D. \& JAYNE, M. The creative countryside: Policy and practice in the UK rural cultural economy. Journal of Rural Studies, 2010, № 26, p. 209-218.

BERTACCHINI, E. \& BORRIONE, P. The Geography of the Italian Creative Economy: The Special Role of the Design and Craft-based Industries. Regional Studies, 2013, Vol. 47, No 2, p. 135-147.

BOAL, I, y HERRERO, L.C. La distribución espacial de las actividades culturales y creativas en Castilla y León un análisis mediante técnicas de econometría espacial. Alcalá de Henares: Universidad de Alcalá de Henares, Seminario Internacional sobre Economía Regional de las Actividades Creativas y los Servicios Innovadores, 2015.

BOIX, R.; LAZERETTI, L.; HERVÁS, J.L. \& DE MIGUEL, B. Creative clusters in Europe. A microdata approach. European Regional Science Association. ERSA conference paper, 2011, No 119471.

BOIX, R. \& LAZZERETTI, L. Creative industries in Spain: A panorama. Investigaciones Regionales, 2012, No 22, p. 181-206.

CHAPAIN, C.; COOKE, P.; DE PROPRIS, L.; MACNEILL, S. \& MATEOS-GARCÍA, J. Creative clusters and innovation. Putting creativity on the map. Londres: NESTA, 2010.

CROMLEY, R. \& HANINK, D. Focal Location Quotients: Specification and Applications. Geographical analysis, 2012, Vol. 44, № 4, p. 398-410.

DE PROPRIS, L.; CHAPAIN, C.; COOKE, P.; MACNEIL, S. \& MATEOS, M. The Geography of Creativity. Londres: NESTA, 2009.

ENDERS, A. \& HAYE, C. Europe's creative hubs. Londres: Bertelsmann, 2014. 
ESCALONA-ORCAO, A.I.; ESCOLANO-UTRILLA, S.; FRUTOS-MEJÍAS, L.M.; LOSCERTALES-PALOMAR, B.; SÁEZ-PÉREZ, L.A. \& SÁNCHEZ-VALVERDE GARCÍA, B. A pathway for local development. The clusters of creative industries in small cities and rural areas of Spain. Semestrale di Studi e Ricer- che di Geografia, 2015, No 1, p. 51-62.

ESCALONA-ORCAO, A.I.; ESCOLANO-UTRILLA, S.; SÁEZ-PÉREZ, L.A. \& SÁNCHEZ-VALVERDE GARCÍA, B. The location of creative clusters in non-metropolitan areas: A methodological proposition. Journal of Rural Studies, 2016, No 45, p. 112-122.

EUROPEAN COMMISSION. Policy handbook on how strategically use the EU support programes, including structural funds, to Foster the potential of culture for local, regional and national development and the spill-overs effects on the wider economy. 2012.

JAKOB, D. \& VAN HEUR, B. Editorial: taking mateers into thirds hands: intermediaries and the organization of the creative economy. Regional Studies, 2015, Vol. 49, No 3, p. 357-361.

LAFUENTE, E.; VAILLANT, Y. \& SERAROLS, C. Location decisions of knowledge-based entrepreneurs: why some Catalan KISAs choose to be rural? Technovation, 2010, Vol. 30, No 11-12, p. 590-600.

LAZZERETTI, L.; BOIX, R. \& CAPONE, F. Do creative industries cluster? Mapping creative local production systems in Italy and Spain. Industrial Innovation, 2008, Vol. 15, No 5, p. 549-567.

MATEOS GARCIA, J; BAKHSHI, H. \& LENEL, M. A map of the videogames industry. Londres: NESTA, 2014.

MCGRANAHAN, D. \& WOJAN, T. Recasting the creative class to examine growth processes in rural and urban counties. Regional Studies, 2007, Vol. 41, No 2, p. 197-216.

MCGRANAHAN, D.; WOJAN, T. \& LAMBERT, D. The rural growth trifecta: outdoor amenities, creative class and entrepreneurial context. Journal of Economic Geography, 2010, Vol. 11, No 3, p. 529-557.

MCCANN, P. \& ORTEGA-ARGILÉS, R. Smart specialization, regional growth and applications to European Union cohesion policy. Regional Studies, 2015, No 49, Vol. 8, p. 1291-1302.

MÉNDEZ, R.; MICHELINI, J.J.; PRADA, J. \& TÉBAR, J. Economía creativa y desrrollo urbano en España. Una aproximación a sus lógicas espaciales. EURE, 2012, Vol. 38, № 113, p. 5-32.

MINISTERIO DE FOMENTO. Atlas Estadístico de las Áreas Urbanas en España, 2006. Disponible en Internet: http://atlas.vivienda.es/

MORENO JIMÉNEZ, A. Modelización cartográfica de densidades mediante estimadores Kernel. Treballs de la Societat Catalana de Geografia, 1991, № 30, p. 155-170.

NALDI, L.; NILSSON, P.; WESTLUND, H. \& WIXE, S. What is smart rural development? Journal of Rural Studies, Vol. 40, p. 90-101.

PORTER, M.E. Clusters and the new economics of competition. Harvard Business Review, 1998, p. 77-90. 
RODRÍGUEZ-POSE, A. \& COMPTOUR, F. Do clusters generate greater innovation and growth? An analysis of European regions. Professional Geographer, 2012, Vol. 64, № 2, p. 211-231.

SPENCER, G.M.; VINODRAI, T.: GERTLER, M.S. \& WOLFE, D.A. Do clusters make a difference? Defining and assessing their economic performance. Regional Studies, 2010, Vol. 44, No 6, p. 697-715.

TITZE, M.; BRACHERT, M. \& KUBIS, A. Actors and interactions-identifying the role of industrial clusters for regional production and knowledge generation activities. Growth \& Change, 2014, Vol $45, N^{\circ} 2$, p. 163-190.

UNCTAD (UNITED NATIONS CONFERENCE FOR TRADE AND DEVELOPMENT). Creative Economy Report 2010. Ginebra. Disponible en Internet: http://unctad.org/es/Docs/ditctab20103_en.pdf

VALLANCE, P. Creative knowing, organisational learning, and socio-spatial expansion in UK videogame development studios. Geoforum, 2014, № 51, p. 15-26.

VILLARREAL GONZÁLEZ, A. \& FLORES SEGOVIA, M. Identificación de clusters espaciales y su especialización económica en el sector de innovación. Región y Sociedad, 2015, № 62, p. 117-147.

ZÚNIIGA, M., PUEYO, A. \& CALVO, J. The Spanish population during the twentieth century and beyond. Journal of Maps, 2012, Vol. 8, No 4, p. 386-391. 
